\title{
Projected BCS states and spin Hamiltonians for the $\mathrm{SO}(n)_{1}$ WZW model
}

\author{
Hong-Hao $\mathrm{Tu}$ \\ Max-Planck-Institut für Quantenoptik, Hans-Kopfermann-Str. 1, D-85748 Garching, Germany
}

(Dated: October 30, 2018)

\begin{abstract}
We propose a class of projected BCS wave functions and derive their parent spin Hamiltonians. These wave functions can be formulated as infinite Matrix Product States constructed by chiral correlators of Majorana fermions. In 1D, the spin Hamiltonians can be viewed as $\mathrm{SO}(n)$ generalizations of Haldane-Shastry models. We numerically compute the spin-spin correlation functions and Rényi entropies for $n=5$ and 6 . Together with the results for $n=3$ and 4 , we conclude that these states are critical and their low-energy effective theory is the $\mathrm{SO}(n)_{1}$ Wess-Zumino-Witten model. In 2D, we show that the projected BCS states are chiral spin liquids, which support non-Abelian anyons for odd $n$ and Abelian anyons for even $n$.
\end{abstract}

PACS numbers: 75.10.Pq, 11.25.Hf, 03.65.Fd

Introduction.- An efficient description of quantum many-body systems is a challenging problem in modern physics, as the dimension of the Hilbert space scales exponentially with the number of particles. For strongly interacting many-body systems, much of our understanding of their properties comes from physically motivated trial wave functions and/or exact solutions of specific models. A great success of the trial wave function approach is the celebrated Laughlin wave function for the fractional quantum Hall effect at $1 / m$ (with $m$ odd) filling [1]. Toward exact results, Bethe's solution of the spin$1 / 2$ Heisenberg chain [2] and integrability of the spin-1/2 Haldane-Shastry model [3] provide invaluable insight for critical spin- $1 / 2$ chains.

The justification of trial wave functions is usually a difficult task. For example, the relevance of Anderson's resonating valence bond (RVB) state [4] for the mechanism of high- $T_{c}$ superconductivity is still a controversial issue. A useful technique for justifying trial wave functions is to study their parent Hamiltonians for which the trial wave functions are exact ground states. For the Laughlin wave function, the parent Hamiltonian which consists of certain Haldane pseudopotentials [5] differs from physical Coulomb interactions but their difference can be viewed as a perturbation [6]. A similar situation arises for the spin-1 Affleck-Kennedy-Lieb-Tasaki (AKLT) state and its parent Hamiltonian [7], which contains an extra biquadratic term apart from Heisenberg interactions. Since the spin-1 AKLT model can be adiabatically connected to the spin-1 Heisenberg chain without closing the gap, it is widely believed that the AKLT state qualitatively captures the physics of the spin-1 Heisenberg chain.

In this work, we propose a class of projected BCS states and derive their parent Hamiltonians. These states can also be represented as infinite Matrix Product States (MPSs) 8] constructed from chiral correlators of Majorana fermions. In $1 \mathrm{D}$, the spin Hamiltonians are $\mathrm{SO}(n)$ generalizations of Haldane-Shastry models. We numerically calculate the spin-spin correlation functions and the Rényi entropies for $n=5$ and 6 and compare the nu- merical results with field theory predictions from $\mathrm{SO}(n)_{1}$ criticality. Together with the known results for $n=3$ and 4 , we expect that for general $n$ these states are critical and belong to the $\mathrm{SO}(n)_{1}$ Wess-Zumino-Witten (WZW) universality class. We also show that the projected BCS states with modified Cooper pair wave functions provide a good description for Ising ordered and disordered phases close to $\mathrm{SO}(n)_{1}$ criticality. In $2 \mathrm{D}$, the projected BCS states are chiral spin liquids with $p+i p$ pairing symmetry. We find that these topological states support non-Abelian Ising anyons for odd $n$ and Abelian anyons for even $n$, respectively.

Projected BCS wave function.- Constructing the projected BCS wave functions relies on a slave-particle representation of the $\mathrm{SO}(n)$ algebra. Let us start from a $1 \mathrm{D}$ periodic chain with even $N$ sites, where the $n$ vectors in each site are represented by using singly occupied fermions, $\left|n^{a}\right\rangle=c_{a}^{\dagger}|0\rangle(a=1, \ldots, n)$. In terms of fermions, the $\mathrm{SO}(n)$ generators are written as $L^{a b}=i\left(c_{a}^{\dagger} c_{b}-c_{b}^{\dagger} c_{a}\right)$, where $1 \leq a<b \leq n$. To remove unphysical states in this fermionic representation, a single-occupancy constraint is required, $\sum_{a=1}^{n} c_{j, a}^{\dagger} c_{j, a}=1 \forall j=1, \ldots, N$, which defines a Gutzwiller projector $P_{\mathrm{G}}$. Then, the projected BCS wave function of our interest is defined by

$$
|\Psi\rangle=P_{\mathrm{G}} \exp \left(\sum_{i<j} \frac{1}{z_{i}-z_{j}} \sum_{a=1}^{n} c_{i, a}^{\dagger} c_{j, a}^{\dagger}\right)|0\rangle,
$$

where $\sum_{a=1}^{n} c_{i, a}^{\dagger} c_{j, a}^{\dagger}$ creates an $\mathrm{SO}(n)$ singlet between sites $i$ and $j$. Note that $|\Psi\rangle$ is a coherent superposition of valence-bond singlets of arbitrary range (see Fig. 1) and hence can be viewed as an RVB state [4]. If we choose $z_{j}=\exp \left(i \frac{2 \pi}{N} j\right)$, the amplitude of the Cooper pair wave function $1 /\left|z_{i}-z_{j}\right|$ is the inverse of the chord distance between the sites. Under this choice, $|\Psi\rangle$ is both real and translationally invariant, which is the uniform case that we will consider in the following.

Before discussing the properties of $|\Psi\rangle$ for general $n$, we establish the relation between (11) and some known results. For $n=3$, after switching to the standard spin-1 


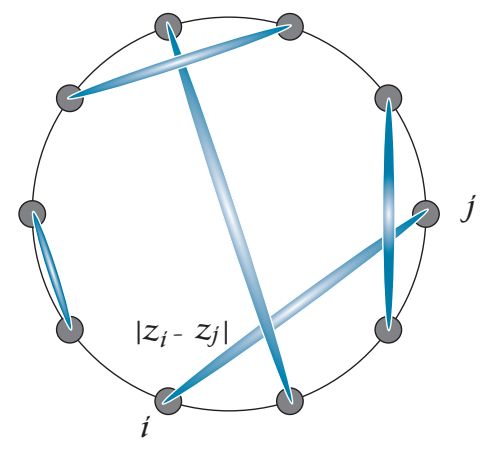

FIG. 1: (Color online) Schematic of a valence-bond configuration in the projected BCS state (1). The valence bonds (blue) are $\mathrm{SO}(n)$ singles formed by two $\mathrm{SO}(n)$ vectors. In the uniform case $z_{j}=\exp \left(i \frac{2 \pi}{N} j\right)$, the periodic chain is viewed as a unit circle and $\left|z_{i}-z_{j}\right|$ is the chord distance between two sites.

basis $\left|n^{1}\right\rangle=\frac{1}{\sqrt{2}}(|-1\rangle-|1\rangle),\left|n^{2}\right\rangle=\frac{i}{\sqrt{2}}(|-1\rangle+|1\rangle)$, $\left|n^{3}\right\rangle=|0\rangle$, we find that the projected BCS state (10) is equivalent to the spin-1 Haldane-Shastry state, which has been considered in Refs. [9 11]. It was shown [9, 10] that this state is critical and its low-energy effective theory is an $\mathrm{SU}(2)_{2}$ (or equivalently $\left.\mathrm{SO}(3)_{1}\right)$ WZW model. In a recent work [12], a projected BCS wave function similar to (1) is used as a variational ansatz to describe the phases in the spin-1 bilinear-biquadratic chain, including the Takhtajan-Babujian model [13] which also belongs to the $\mathrm{SU}(2)_{2}$ WZW universality class [14]. For $n=4$, after representing the four $\mathrm{SO}(4)$ vectors by two spin- $1 / 2$ states, we find that the projected BCS state (11) can be rewritten as a product of two decoupled spin- $1 / 2$ Haldane-Shastry states. An immediate consequence of this decomposition is that the $\mathrm{SO}(4)$ state is critical and represents the fixed point of the $\mathrm{SO}(4)_{1}$ WZW model.

Infinite MPS and parent Hamiltonian.- From the known results for $n=3$ and 4 , one may speculate that for general $n$ the projected BCS state (1) belongs to the $\mathrm{SO}(n)_{1}$ WZW universality class. Let us further uncover this relationship by formulating (11) as an infinite MPS. The $\mathrm{SO}(n)_{1}$ WZW model has a primary field with conformal weight $h_{v}=1 / 2$ in the vector representation [15], which can be naturally interpreted as Majorana fermion fields $\chi^{a}(z)(a=1, \ldots, n)$. This Majorana representation of the primary field allows us to rewrite the projected BCS state (1) as the following infinite MPS:

$$
|\Psi\rangle=\sum_{a_{1}, \ldots, a_{N}=1}^{n} \Psi\left(a_{1}, \ldots, a_{N}\right)\left|n^{a_{1}}, \ldots, n^{a_{N}}\right\rangle,
$$

where the coefficients are the chiral correlators of $N$ Majorana fields [16]

$$
\Psi\left(a_{1}, \ldots, a_{N}\right)=\left\langle\chi^{a_{1}}\left(z_{1}\right) \chi^{a_{2}}\left(z_{2}\right) \cdots \chi^{a_{N}}\left(z_{N}\right)\right\rangle .
$$

A detailed proof of the equivalence of the projected BCS state (11) and the infinite MPS (3) is given in the Supplemental Material [17].

Unlike usual MPS with finite matrix dimensions, the state (3) is an infinite MPS [8] since its ancillary space on which the Majorana fields act is an infinite-dimensional Hilbert space. This allows the infinite MPS (3) to describe the expected $\mathrm{SO}(n)_{1}$ criticality with unbounded increase of the entanglement entropy.

The key benefit of the infinite MPS formulation is that a parent Hamiltonian can be derived, such that (3) is its exact ground state. As shown in Ref. [9], the presence of null vectors in conformal field theories (CFTs) leads to a set of operators which annihilate the infinite MPS. Following this approach, we have derived [17] such operators for (3)

$\Lambda_{i}^{a b}=\sum_{j(\neq i)} \frac{w_{i j}}{3}\left[2 L_{j}^{a b}-\frac{3}{n-1} L_{i}^{a b}\left(\vec{L}_{i} \cdot \vec{L}_{j}\right)+\left(\vec{L}_{i} \cdot \vec{L}_{j}\right) L_{i}^{a b}\right]$, where $w_{i j} \equiv\left(z_{i}+z_{j}\right) /\left(z_{i}-z_{j}\right)$ and $\vec{L}_{i} \cdot \vec{L}_{j} \equiv \sum_{a<b} L_{i}^{a b} L_{j}^{a b}$. Since $\Lambda_{i}^{a b}|\Psi\rangle=0 \forall i, a, b$ and $\sum_{i} L_{i}^{a b}|\Psi\rangle=0 \forall a, b$, we can define a parent Hamiltonian $H=\sum_{i, a<b}\left(\Lambda_{i}^{a b}\right)^{\dagger} \Lambda_{i}^{a b}+$ $\frac{2(N-2)}{3} \sum_{a<b}\left(\sum_{i} L_{i}^{a b}\right)^{2}+E_{0}$ whose ground state is the infinite MPS (3) with energy $E_{0}$. Choosing $E_{0}=-\frac{2}{9}(n-$ 1) $N\left(N^{2}-4\right)$, the explicit form of $H$ is given by

$$
\begin{aligned}
H= & -\sum_{i \neq j} w_{i j}^{2}\left[\frac{n+2}{3}\left(\vec{L}_{i} \cdot \vec{L}_{j}\right)+\frac{n-4}{3(n-1)}\left(\vec{L}_{i} \cdot \vec{L}_{j}\right)^{2}\right] \\
& -\frac{n-4}{3(n-1)} \sum_{i \neq j \neq k} w_{i j} w_{i k}\left(\vec{L}_{i} \cdot \vec{L}_{j}\right)\left(\vec{L}_{i} \cdot \vec{L}_{k}\right) .
\end{aligned}
$$

Generically, the Hamiltonian (5) is a long-ranged $\mathrm{SO}(n)$ bilinear-biquadratic model with three-spin interactions. For $n=4$, as we expected, the Hamiltonian only has inverse-square Heisenberg exchange interactions, which can be decomposed into two spin-1/2 Haldane-Shastry Hamiltonians due to $\mathrm{SO}(4) \simeq \mathrm{SU}(2) \times \mathrm{SU}(2)$.

Jastrow versus Pfaffian.- It is well known that the $\mathrm{SO}(n)$ Lie algebra has a sharp difference between even and odd $n$ [18]. As we shall see, this leads to distinct forms of the wave function (1) in the Cartan basis for even and odd $n$ : The former has a pure Jastrow form, while the latter includes a Pfaffian factor. To see this difference, let us consider $\mathrm{SO}(2 l)$ and $\mathrm{SO}(2 l+1)$ ( $l$ : integer) and choose mutually commuting Cartan generators as $L^{12}, L^{34}, \ldots L^{2 l-1,2 l}$. For $\mathrm{SO}(2 l)$, a convenient choice of the Cartan basis is defined by $\left|0, \ldots, m_{\alpha}= \pm 1, \ldots, 0\right\rangle=$ $\left(\left|n^{2 \alpha}\right\rangle \pm i\left|n^{2 \alpha-1}\right\rangle\right) / \sqrt{2}(\alpha=1, \ldots, l)$, where $m_{\alpha}$ is the eigenvalue of $L^{2 \alpha-1,2 \alpha}$. For the vectors $\mid 0, \ldots, m_{\alpha}=$ $\pm 1, \ldots, 0\rangle$, we label their positions in the spin chain by $x_{1}^{(\alpha)}<\cdots<x_{N_{\alpha}}^{(\alpha)}$. In this basis, the wave function (11) for even $n=2 l$ takes the form [17]

$$
\Psi(\{m\})=\rho_{m} \prod_{\alpha=1}^{l} \prod_{i<j}\left(z_{i}-z_{j}\right)^{m_{\alpha, i} m_{\alpha, j}},
$$


where $\rho_{m}=\operatorname{sgn}\left(x_{1}^{(1)} \ldots x_{N_{1}}^{(1)} \ldots x_{1}^{(l)} \ldots x_{N_{l}}^{(l)}\right)$ (sgn: signature of a permutation) if $\sum_{i} m_{\alpha, i}=0 \forall \alpha$ and $\rho_{m}=0$ otherwise.

For $\mathrm{SO}(2 l+1)$, apart from the vectors $\mid 0, \ldots, m_{\alpha}=$ $\pm 1, \ldots, 0\rangle$, there exists an additional vector $|0, \ldots, 0\rangle=$ $\left|n^{2 l+1}\right\rangle$, which is annihilated by all Cartan generators. Labeling their positions by $x_{1}^{(0)}<\cdots<x_{N_{0}}^{(0)}$, the wave function (11) for odd $n=2 l+1$ is written as [17]

$$
\Psi(\{m\})=\rho_{m} \operatorname{Pf}_{0}\left(\frac{1}{z_{i}-z_{j}}\right) \prod_{\alpha=1}^{l} \prod_{i<j}\left(z_{i}-z_{j}\right)^{m_{\alpha, i} m_{\alpha, j}},
$$

where $\rho_{m}=\operatorname{sgn}\left(x_{1}^{(0)} \ldots x_{N_{0}}^{(0)}, x_{1}^{(1)} \ldots x_{N_{1}}^{(1)} \ldots x_{1}^{(l)} \ldots x_{N_{l}}^{(l)}\right)$ if $\sum_{i} m_{\alpha, i}=0 \forall \alpha$ and $\rho_{m}=0$ otherwise, and the Pfaffian factor $\operatorname{Pf}_{0}\left(\frac{1}{z_{i}-z_{j}}\right)$ is restricted to the positions for the extra vector $|0, \ldots, 0\rangle$.

Numerical results.- The power-law decaying correlation functions and the universal scaling of entanglement entropy [19] are characteristic behaviors of conformal critical points in 1D. Even though these quantities are difficult to compute analytically for (11), the Jastrow and Pfaffian forms (6) and (7) of the wave functions are very suitable for determining them numerically via the Metropolis Monte Carlo (MC) method [20]. Below we focus on the projected BCS state (1) with $n=5$ and 6 and provide numerical evidence that they belong to $\mathrm{SO}(5)_{1}$ and $\mathrm{SO}(6)_{1}$ WZW models, respectively.

For critical spin chains in the $\mathrm{SO}(n)_{1}$ WZW universality class, field theory predicts that for $n<8$ the spin-spin correlation function behaves as $\left\langle L_{j}^{a b} L_{j+x}^{a b}\right\rangle \sim(-1)^{x} / x^{\eta}$ with $\eta=n / 4$ [21]. For the projected BCS state (11) with $n=5$ and 6 , we have computed the two-point spin correlator $\left\langle L_{j}^{12} L_{j+x}^{12}\right\rangle$. Figure 2 shows the numerical results for $N=200$. The critical exponents that best fit with our numerical data are $\eta=1.22$ for $\mathrm{SO}(5)$ and $\eta=1.42$ for $\mathrm{SO}(6)$ (solid lines in Fig. 2), which agree very well with the field theory predictions (dotted lines).

The entanglement entropy that is easily accessible via the MC method is the Rényi entropy $S_{L}^{(2)}=-\ln \operatorname{Tr} \rho_{L}^{2}$ (see Refs. [8, 22 24]), where $\rho_{L}$ is the reduced density matrix of the state in a subsystem of length $L$. For the $\mathrm{SO}(n)_{1}$ WZW model with $c=n / 2$ we expect $S_{L}^{(2)}=$ $c \ln [\sin (\pi L / N)] / 4+c_{2}^{\prime}$ [19], where $c_{2}^{\prime}$ is a constant. For $n=5$ and 6 , we plot $S_{L}^{(2)}$ as a function of $\ln [\sin (\pi L / N)] / 4$ for $N=200$ in Fig. 3. From our MC data, the estimates of the central charge are $c=2.31$ for $\mathrm{SO}(5)$ and $c=$ 2.76 for $\mathrm{SO}(6)$ (solid lines in Fig. 3), which are close to the predicted $c=n / 2$ (dotted lines) but show some deviations.

The origin of the small deviations of the numerical results and the $\mathrm{SO}(n)_{1}$ predictions may be due to the presence of marginally irrelevant terms in the $\mathrm{SO}(n)_{1}$ WZW model for (11) and its parent Hamiltonian (5), unlike the $\mathrm{SU}(n)$ Haldane-Shastry models [25, 26] (including the spin-1/2 Haldane-Shastry model for $n=2$ ) being the
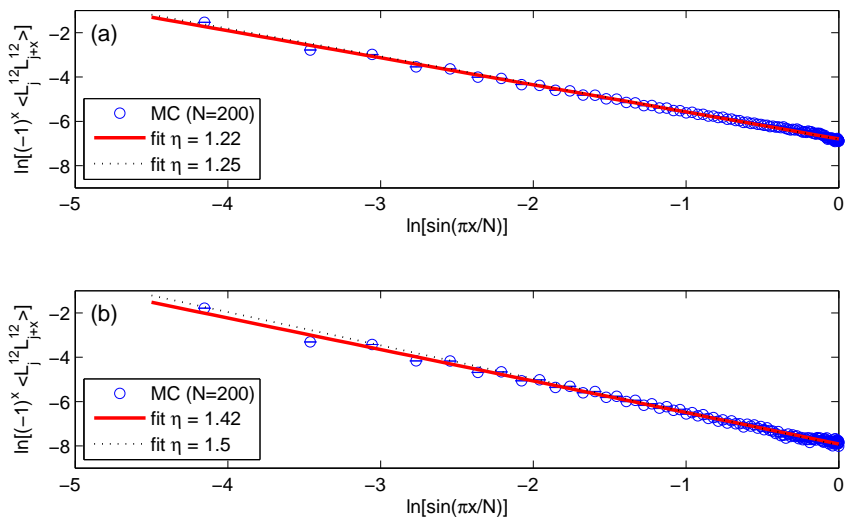

FIG. 2: (Color online) Spin-spin correlation function (logarithmic scale) $\ln \left[(-1)^{x}\left\langle L_{j}^{12} L_{j+x}^{12}\right\rangle\right]$ as a function of $\ln [\sin (\pi x / N)]$ in the projected BCS state (11) for $N=200$ and (a) $n=5$ and (b) $n=6$. The solid lines (red) are fits of the form $\ln \left[(-1)^{x}\left\langle L_{j}^{12} L_{j+x}^{12}\right\rangle\right]=\eta \ln [\sin (\pi x / N)]+A$, where $\eta$ and $A$ are fitting parameters. The dotted lines are also fits of this formula but with the field theory prediction $\eta=n / 4$ (Ref. [21]).
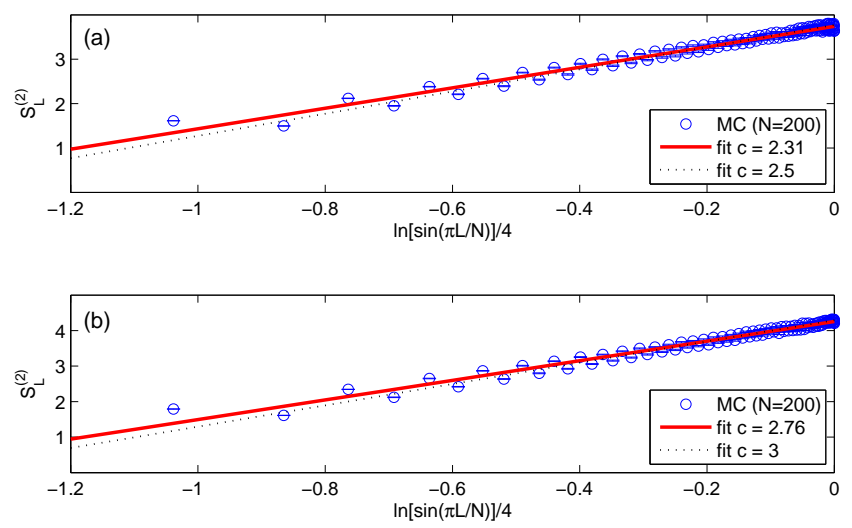

FIG. 3: (Color online) Rényi entropy $S_{L}^{(2)}$ as a function of $\ln [\sin (\pi L / N)] / 4$ in the projected BCS state (1) for $N=200$ and (a) $n=5$ and (b) $n=6$. The solid lines (red) are fits of the form $S_{L}^{(2)}=c \ln [\sin (\pi L / N)] / 4+c_{2}^{\prime}$, where $c$ and $c_{2}^{\prime}$ are fitting parameters. The dotted lines are also fits of this formula but the central charge $c$ is fixed to $c=n / 2$ of the $\mathrm{SO}(n)_{1}$ WZW model.

fixed points of the $\mathrm{SU}(n)_{1}$ WZW model. For $n=3$, the presence of marginal term in the spin-1 Haldane-Shastry model has been confirmed numerically [9, 10]. If this is also the case for $n \geq 5$, an interesting open question is whether there exist a modified version of (1) and its parent Hamiltonian that represent the fixed point of the $\mathrm{SO}(n)_{1}$ WZW model.

Away from $S O(n)_{1}$ criticality.- After showing that the projected BCS state (1) captures the physics of the $\mathrm{SO}(n)_{1}$ WZW model, it is natural to ask whether similar projected wave functions are relevant for gapped spin chains away from (but close to) $\mathrm{SO}(n)_{1}$ criticality. Let us restrict ourselves to $\mathrm{SO}(n)$ symmetric models for sim- 
plicity. According to the well-known result by Witten [27], the $\mathrm{SO}(n)_{1} \mathrm{WZW}$ model is equivalent to $n$ massless Majorana fermions, i.e., $n$ Ising models at criticality. For this critical theory, the only relevant perturbation allowed by $\mathrm{SO}(n)$ symmetry is the mass term of Majorana fermions. Thus, the low-energy effective theory has the Hamiltonian density $\mathcal{H}=-\frac{i v}{2} \sum_{\nu=1}^{n}\left(\xi_{R}^{\nu} \partial_{x} \xi_{R}^{\nu}-\xi_{L}^{\nu} \partial_{x} \xi_{L}^{\nu}\right)-$ $i m \sum_{\nu=1}^{n} \xi_{R}^{\nu} \xi_{L}^{\nu}$, where $\xi_{R(L)}^{\nu}$ are right (left) moving Majorana fermions, $v$ and $m$ are their velocity and mass. Here we have assumed four-fermion interactions are weak and can be neglected, since they are marginal and only renormalize the mass of Majorana fermions at low-energy limit [28].

The $\mathrm{SO}(n)_{1}$ criticality corresponds to $m=0$. The two gapped phases adjacent to the $\mathrm{SO}(n)_{1}$ criticality are (i) the Ising ordered phase $(m<0)$ and (ii) the Ising disordered phase $(m>0)$. For these two phases, we note that they can be well described by modified projected BCS states. Actually, these two gapped phases and an $\mathrm{SO}(n)_{1}$ critical point (Reshetikhin model [29]) are realized in the $\mathrm{SO}(n)$ bilinear-biquadratic chain [21, 30]. The ideal example that belongs to the Ising ordered phase is the $\mathrm{SO}(n)$ AKLT model [7, 31, 32], whose ground state can be represented as a projected BCS state, by replacing $g_{i j}=1 /\left(z_{i}-z_{j}\right)$ in (1) with $g_{i j}=1$ [12]. For the Ising disordered phase, the ground state of the spin chain is dimerized [21] and hence the valence bonds are short ranged. In this case, a proper Cooper pair wave function for the projected BCS state can be chosen as $g_{i j} \sim \exp \left(-\left|z_{i}-z_{j}\right| / \xi\right)$, where $\xi$ is the length scale of the valence bonds. In the extreme case, a Cooper pair wave function that is nonvanishing only between neighboring sites yields a Majumdar-Ghosh-like state, corresponding to perfect dimerization. These results imply that both Ising ordered and disordered phases close to $\mathrm{SO}(n)_{1}$ criticality are well described by projected BCS states with properly chosen $g_{i j}$. Indeed, for $n=3$, it was shown [12] that the projected BCS states with Cooper pair wave functions generated from Kitaev's Majorana chains [33] are good variational wave functions for the Haldane (Ising ordered) and the dimerized (Ising disordered) phases.

$2 D$ chiral spin liquids. - After establishing the relevance of projected BCS states (1) for $\mathrm{SO}(n)_{1}$ criticality in 1D, we move on and discuss their properties in a $2 \mathrm{D}$ square lattice, where the $z$ 's in (3) are now complex coordinates of the lattice sites. In an analogy with fractional quantum Hall (FQH) states constructed by conformal blocks of their gapless edge CFTs [34, 35], the chiral correlator (3) from the $\mathrm{SO}(n)_{1}$ WZW model ( $n$ massless Majorana fermions) yields chiral spin liquids, which break time reversal symmetry and are spin counterparts of FQH states [36]. From the projected BCS form (11), the Cooper pair wave function $1 /\left(z_{i}-z_{j}\right)$ now corresponds to the topological phase of $p+i p$ superconductors [37] supporting chiral gapless Majorana edge modes, which justifies the above bulk-edge correspondence. Below we focus on the anyonic quasiparticle excitations in these 2D states, which have intriguing properties depending on $n \bmod 16$, i.e., a 16-fold way.

For odd $n$, the quasiparticles built upon the $\mathrm{SO}(n)$ states support non-Abelian statistics. Let us adapt the CFT approach of creating quasihole excitations in FQH states [34] to our spin system. For odd $n$, the $\operatorname{SO}(n)_{1}$ WZW model has three primary fields: identity field $I$, vector field $v$, and spinor field $s$. Following the CFT approach, creating quasiparticles in the $\mathrm{SO}(n)$ state is achieved by adding spinor fields $s$ in the chiral correlator (3). Then, the statistics of quasiparticles are encoded in the fusion rules of the primary fields. In fact, the spinor fields have a nontrivial fusion rule $s \times s=I+v$, together with $s \times v=s$ and $v \times v=I$. These fusion rules resemble those in Ising CFT $(\sigma \times \sigma=I+\varepsilon, \sigma \times \varepsilon=\sigma$, and $\varepsilon \times \varepsilon=I$ ), which are responsible for the non-Abelian statistics of Ising anyons [38]. Indeed, the Majorana free field representation of $\mathrm{SO}(n)_{1}$ WZW model allows us to identify the spinor fields $s$ with conformal weight $h_{s}=$ $n / 16$ as a product of $n$ Ising $\sigma$ fields $\left(h_{\sigma}=1 / 16\right)$. Thus, we conclude that the $\mathrm{SO}(n)$ states support non-Abelian Ising anyons for odd $n$. Note that the case with $n=3$ recovers the physics of the Moore-Read states [34, 39], while for odd $n \geq 5$ they are natural generalizations of the Moore-Read states.

Now we show that the $\mathrm{SO}(n)$ states only support Abelian anyons for even $n$. This subtle difference roots in the fusion rules of the $\mathrm{SO}(n)_{1}$ primary fields. In contrast to odd $n$ case, the $\mathrm{SO}(n)_{1}$ WZW model with even $n$ has two spinor primary fields $s_{+}$and $s_{-}$with conformal weight $h_{s_{+}}=h_{s_{-}}=n / 16$ [15], apart from the usual identity and vector fields. The fusion rules of spinor and vector fields are $s_{+} \times v=s_{-}$and $s_{-} \times v=s_{+}$. Depending on the parity of $n / 2$, the fusion rules involving two spinor fields are $s_{+} \times s_{+}=s_{-} \times s_{-}=I, s_{+} \times s_{-}=v$ for even $n / 2$ and $s_{+} \times s_{+}=s_{-} \times s_{-}=v, s_{+} \times s_{-}=I$ for odd $n / 2$ [40]. However, due to the absence of multiplicity in the fusion outcome, only Abelian anyons can exist in the $\mathrm{SO}(n)$ states with even $n$.

More precisely, the anyonic properties of the $\mathrm{SO}(n)$ states depend on $n \bmod 16$ (16-fold way) [41]. The topological spin of the quasiparticles generated by $\mathrm{SO}(n)$ spinor primary fields is $\theta_{s}=e^{i 2 \pi h_{s}}=e^{i n \pi / 8}$ (for both odd and even $n$ ), which is a clear signature of the 16-fold way. For example, the quasiparticles $s_{+}$ and $s_{-}$for $\mathrm{SO}(8)$ have $\theta_{s_{+}}=\theta_{s_{-}}=-1$, which are both fermions. Actually, this 16-fold way of the anyonic properties has been analyzed in detail by Kitaev. In Ref. [38], he considered a theory with $Z_{2}$ vortices and free Majorana fermions whose energy band has Chern number $\nu$ and showed that the anyonic properties of the unpaired Majorana modes in the vortex core depends on $\nu \bmod$ 16. Thus, our present work shows that the $\mathrm{SO}(n)_{1} \mathrm{CFT}$ is responsible for this 16 -fold way and provides a class of 
microscopic Hamiltonians which realize this interesting physics.

Conclusion and perspective.- To conclude, we have proposed a class of projected BCS states and derived their parent Hamiltonians. These states also have an infinite MPS form generated by chiral correlators of Majorana fermions. In $1 \mathrm{D}$, they can be viewed as $\mathrm{SO}(n)$ generalizations of Haldane-Shastry models and capture the physics of the $\mathrm{SO}(n)_{1}$ WZW model. These results indicate that modified projected BCS states are good variational ansatz for describing Ising ordered and disordered phases close to $\mathrm{SO}(n)_{1}$ criticality. In $2 \mathrm{D}$, the $\mathrm{SO}(n)$ states are chiral spin liquid states, which support non-Abelian Ising anyons for odd $n$ and Abelian anyons for even $n$. An open question that deserves further investigation is whether these $2 \mathrm{D}$ chiral spin liquids are relevant for physical models and materials [42]. Moreover, our 2D toy models may also shed light on another interesting open question: Can $p+i p$ pairing states arise after doping these antiferromagnets?

The author is indebted to G. Sierra for numerous helpful comments, for sharing his broad knowledge, and also for a careful reading of the manuscript, and sincerely thanks J. I. Cirac for enlightening guidance and encouragement. He is also grateful to Z.-X. Liu, Y. Zhou, X.-G. Wen, T.-K. Ng, and R. Orús for collaborations on related topics, and F. Mezzacapo, A. E. B. Nielsen, M. Cheng, X.-L. Qi, and A. Müller-Hermes for stimulating discussions. This work has been supported by the EU project AQUTE.

[1] R. B. Laughlin, Phys. Rev. Lett. 50, 1395 (1983).

[2] H. Bethe, Z. Phys. 71, 205 (1931).

[3] F. D. M. Haldane, Phys. Rev. Lett. 60, 635 (1988); B. S. Shastry, ibid. 60, 639 (1988).

[4] P. W. Anderson, Science 235, 1196 (1987).

[5] F. D. M. Haldane, Phys. Rev. Lett. 51, 605 (1983).

[6] F. D. M. Haldane and E. H. Rezayi, Phys. Rev. Lett. 54, 237 (1985).

[7] I. Affleck, T. Kennedy, E. H. Lieb, and H. Tasaki, Phys. Rev. Lett. 59, 799 (1987).

[8] J. I. Cirac and G. Sierra, Phys. Rev. B 81, 104431 (2010).

[9] A. E. B. Nielsen, J. I. Cirac, and G. Sierra, J. Stat. Mech. P11014 (2011).

[10] R. Thomale, S. Rachel, P. Schmitteckert, and M. Greiter, Phys. Rev. B 85, 195149 (2012); M. Greiter, J. Low Temp. Phys. 126, 1029 (2002).

[11] B. Paredes, Phys. Rev. B 85, 195150 (2012).

[12] Z.-X. Liu, Y. Zhou, H.-H. Tu, X.-G. Wen, and T.-K. Ng, Phys. Rev. B 85, 195144 (2012).

[13] L. A. Takhtajan, Phys. Lett. A 87, 479 (1982); H. M. Babujian, ibid. 90, 479 (1982).

[14] I. Affleck, Phys. Rev. Lett. 56, 746 (1986).

[15] P. Di Francesco, P. Mathieu, and D. Sénéchal, Conformal Field Theory (Springer, New York, 1997).

[16] E. Ardonne and G. Sierra, J. Phys. A 43, 505402 (2010).
[17] See Supplemental Material for the proof of the equivalence of projected BCS state and infinite MPS, derivation of the parent Hamiltonian, and derivation of the Jastrow and Pfaffian wave functions in the Cartan basis.

[18] H. Georgi, Lie Algebras in Particle Physics (Perseus Books, Reading, MA, 1999).

[19] C. Holzhey, F. Larsen, and F. Wilczek, Nucl. Phys. B 424, 443 (1994); G. Vidal, J. I. Latorre, E. Rico, and A. Kitaev, Phys. Rev. Lett. 90, 227902 (2003); P. Calabrese and J. Cardy, J. Stat. Mech. P06002 (2004).

[20] P. Horsch and T. A. Kaplan, J. Phys. C 16, L1203 (1983).

[21] H.-H. Tu and R. Orús, Phys. Rev. Lett. 107, 077204 (2011).

[22] M. B. Hastings, I. González, A. B. Kallin, and R. G. Melko, Phys. Rev. Lett. 104, 157201 (2010).

[23] Y. Zhang, T. Grover, and A. Vishwanath, Phys. Rev. Lett. 107, 067202 (2011).

[24] F. Mezzacapo, Phys. Rev. B 86, 045115 (2012).

[25] N. Kawakami, Phys. Rev. B 46, 1005 (1992); 46, 3191 (1992).

[26] Z. N. C. Ha and F. D. M. Haldane, Phys. Rev. B. 46, 9359 (1992).

[27] E. Witten, Commun. Math. Phys. 92, 455 (1984).

[28] A. O. Gogolin, A. A. Nersesyan, and A. M. Tsvelik, Bosonization and Strongly Correlated Systems (Cambridge University Press, Cambridge, England, 1998).

[29] N. Y. Reshetikhin, Theor. Math. Phys. 63, 555 (1985).

[30] A. M. Tsvelik, Phys. Rev. B 42, 10499 (1990); A. A. Nersesyan and A. M. Tsvelik, Phys. Rev. Lett. 78, 3939 (1997); P. Bouwknegt and K. Schoutens, Phys. Rev. Lett. 82, 2757 (1999); F. Alet, S. Capponi, H. Nonne, P. Lecheminant, and I. P. McCulloch, Phys. Rev. B 83, 060407(R) (2011).

[31] H.-H. Tu, G.-M. Zhang, and T. Xiang, Phys. Rev. B 78, 094404 (2008); J. Phys. A 41, 415201 (2008); H.-H. Tu, G.-M. Zhang, T. Xiang, Z.-X. Liu, and T.-K. Ng, Phys. Rev. B 80, 014401 (2009).

[32] A. K. Kolezhuk and H. J. Mikeska, Phys. Rev. Lett. 80, 2709 (1998); D. Scalapino, S. C. Zhang, and W. Hanke, Phys. Rev. B 58, 443 (1998); I. Affleck, D. P. Arovas, J. B. Marston, and D. A. Rabson, Nucl. Phys. B 366, 467 (1991).

[33] A. Kitaev, Phys. Usp. 44, 131 (2001).

[34] G. Moore and N. Read, Nucl. Phys. B 360, 362 (1991).

[35] A. E. B. Nielsen, J. I. Cirac, and G. Sierra, Phys. Rev. Lett. 108, 257206 (2012).

[36] X.-G. Wen, F. Wilczek, and A. Zee, Phys. Rev. B 39, 11413 (1989).

[37] N. Read and D. Green, Phys. Rev. B 61, 10267 (2000).

[38] A. Kitaev, Ann. Phys. (NY) 321, 2 (2006).

[39] M. Greiter and R. Thomale, Phys. Rev. Lett. 102, 207203 (2009).

[40] P. Bouwknegt and K. Schoutens, Nucl. Phys. B 547, 501 (1999).

[41] We thank Xiao-Liang Qi and Meng Cheng for sharing their insights on the 16-fold way of anyonic properties and for explaining Kitaev's results in Ref. [38].

[42] Z.-X. Liu, Y. Zhou, and T.-K. Ng, Phys. Rev. B 81, 224417 (2010); 82, 144422 (2010); M. Serbyn, T. Senthil, and P. A. Lee, Phys. Rev. B 84, 180403(R) (2011); S. Bieri, M. Serbyn, T. Senthil, and P. A. Lee, Phys. Rev. B 86, 224409 (2012). 


\section{Supplemental material}

\section{Equivalence of the projected BCS state and the infinite MPS}

In this Section, we prove the equivalence of the projected BCS state and the infinite MPS.

\section{Projected BCS state}

Let us first expand the projected BCS state

$$
\begin{aligned}
|\Psi\rangle= & P_{\mathrm{G}} \exp \left(\sum_{i<j} \frac{1}{z_{i}-z_{j}} \sum_{a=1}^{n} c_{i, a}^{\dagger} c_{j, a}^{\dagger}\right)|0\rangle \\
= & P_{\mathrm{G}} \prod_{a=1}^{n} \prod_{i<j}\left(1+\frac{1}{z_{i}-z_{j}} c_{i, a}^{\dagger} c_{j, a}^{\dagger}\right)|0\rangle \\
= & P_{\mathrm{G}} \prod_{a=1}^{n}\left[\sum_{N_{a}=0\left(N_{a} \text { even) } x_{1}^{(a)}<\cdots<x_{N_{a}}^{(a)}\right.}^{N} \operatorname{Pf}_{a}\left(\frac{1}{z_{i}-z_{j}}\right) c_{x_{1}^{(a)}, a}^{\dagger} \cdots c_{x_{N_{a}}^{\dagger}, a}^{(a)}\right]|0\rangle \\
= & P_{\mathrm{G}} \sum_{N_{1}, N_{2}, \cdots, N_{n}=0}^{N} \sum_{\left(N_{a} \text { even }\right)} \sum_{x_{1}^{(1)}<\cdots<x_{N_{1}}^{(1)} x_{2}^{(2)}<\cdots<x_{N_{2}}^{(2)}} \cdots \sum_{x_{1}^{(n)}<\cdots<x_{N_{a}}^{(n)}} \prod_{a=1}^{n} \operatorname{Pf}_{a}\left(\frac{1}{z_{i}-z_{j}}\right) \\
& \times\left(c_{x_{1}^{(1)}, 1}^{\dagger} \cdots c_{x_{N_{1}}^{(1)}, 1}^{\dagger}\right)\left(c_{x_{1}^{(2)}, 2}^{\dagger} \cdots c_{x_{N_{2}}^{(2)}, 2}^{\dagger}\right) \cdots\left(c_{x_{1}^{(n)}, n}^{\dagger} \cdots c_{x_{N_{n}}^{(n)}, n}^{(n)}\right)|0\rangle
\end{aligned}
$$

where $N_{a}(a=1, \ldots, n)$ is the number of $c_{a}^{\dagger}$ fermions (i.e. $\left|n^{a}\right\rangle$ vector in the configuration), $x_{1}^{(a)}<\cdots<x_{N_{a}}^{(a)}$ are the positions of $c_{a}^{\dagger}$ fermions in the lattice, and the Pfaffian factor $\operatorname{Pf}_{a}\left(\frac{1}{z_{i}-z_{j}}\right)$ is restricted to the positions for the $c_{a}^{\dagger}$ fermions.

The next step is to implement the Gutzwiller projection. Note that the Gutzwiller projector $P_{\mathrm{G}}$ requires single occupancy. Thus, the positions of fermions $\left(x_{1}^{(1)}<\cdots<x_{N_{1}}^{(1)}, x_{2}^{(2)}<\cdots<x_{N_{2}}^{(2)}, \ldots, x_{1}^{(n)}<\cdots<x_{N_{a}}^{(n)}\right)$ must be all different from each other, so that each site has exactly one fermion. As a result, we have $\sum_{a=1}^{n} N_{a}=N$. After implementing the Gutzwiller projector, we obtain

$$
\begin{aligned}
|\Psi\rangle= & \sum_{N_{1}, N_{2}, \ldots, N_{n}=0}^{N} \sum_{\left(N_{1}+N_{2}+\cdots+N_{n}=N \text { and } N_{a} \text { even }\right)_{\text {all allowed } x_{1}^{(a)}<\cdots<x_{N_{a}}^{(a)}}} \prod_{a=1}^{n} \operatorname{Pf}_{a}\left(\frac{1}{z_{i}-z_{j}}\right) \\
& \times\left(c_{x_{1}^{(1)}, 1}^{\dagger} \cdots c_{x_{N_{1}, 1}^{(1)}, 1}^{\dagger}\right)\left(c_{x_{1}^{(2)}, 2}^{\dagger} \cdots c_{x_{N_{2}, 2}^{(2)},{ }^{(2)}}^{\dagger} \cdots\left(c_{x_{1}^{(n)}, n}^{\dagger} \cdots c_{x_{N_{n}}^{(n)}, n}^{\dagger}\right)|0\rangle\right.
\end{aligned}
$$

The final step is to rearrange the positions of fermionic operators so that they can be identified as a spin state. This rearrangement only results in a sign, depending on the positions of fermions

$$
\begin{aligned}
|\Psi\rangle= & \sum_{N_{1}, N_{2}, \ldots, N_{n}=0}^{N} \sum_{\left(N_{1}+N_{2}+\cdots+N_{n}=N \text { and } N_{a} \text { even }\right) \text { all allowed } x_{1}^{(a)}<\cdots<x_{N_{a}}^{(a)}} \prod_{a=1}^{n} \operatorname{Pf}_{a}\left(\frac{1}{z_{i}-z_{j}}\right) \\
& \times \operatorname{sgn}\left(x_{1}^{(1)}, \ldots, x_{N_{1}}^{(1)}, x_{1}^{(2)}, \ldots, x_{N_{2}}^{(2)}, \ldots, x_{1}^{(n)}, \ldots, x_{N_{n}}^{(n)}\right)\left|x_{1}^{(1)}, \ldots, x_{N_{1}}^{(1)}, x_{1}^{(2)}, \ldots, x_{N_{2}}^{(2)}, \ldots, x_{1}^{(n)}, \ldots, x_{N_{n}}^{(n)}\right\rangle
\end{aligned}
$$

where $\left|x_{1}^{(1)}, \ldots, x_{N_{1}}^{(1)}, x_{1}^{(2)}, \ldots, x_{N_{2}}^{(2)}, \ldots, x_{1}^{(1)}, \ldots, x_{N_{n}}^{(1)}\right\rangle$ is a spin configuration labeled by the positions of the vector $\left|n^{a}\right\rangle$ and $\operatorname{sgn}\left(x_{1}^{(1)}, \ldots, x_{N_{1}}^{(1)}, x_{1}^{(2)}, \ldots, x_{N_{2}}^{(2)}, \ldots, x_{1}^{(n)}, \ldots, x_{N_{n}}^{(n)}\right)$ is the signature of permutation due to the sign factor coming from fermionic anticommutation relations.

Thus, the projected BCS wave function can be written as

$$
\Psi\left(\left\{x^{(1)}\right\},\left\{x^{(2)}\right\}, \ldots\left\{x^{(n)}\right\}\right)=\operatorname{sgn}\left(x_{1}^{(1)}, \ldots, x_{N_{1}}^{(1)}, x_{1}^{(2)}, \ldots, x_{N_{2}}^{(2)}, \ldots, x_{1}^{(n)}, \ldots, x_{N_{n}}^{(n)}\right) \prod_{a=1}^{n} \operatorname{Pf}_{a}\left(\frac{1}{z_{i}-z_{j}}\right)
$$


where $\left\{x^{(a)}\right\}$ is the set of positions satisfying $x_{1}^{(a)}<\cdots<x_{N_{a}}^{(a)}\left(N_{a}\right.$ even and $\left.\sum_{a=1}^{n} N_{a}=N\right)$.

\section{Infinite MPS}

Let us now consider the infinite MPS

$$
|\Psi\rangle=\sum_{a_{1}, \ldots, a_{N}=1}^{n} \Psi\left(a_{1}, \ldots, a_{N}\right)\left|n^{a_{1}}, n^{a_{2}}, \ldots, n^{a_{N}}\right\rangle
$$

where $\Psi\left(a_{1}, \ldots, a_{N}\right)$ are given by the chiral correlators of Majorana fermion fields $\chi^{a}(a=1, \ldots, n)$

$$
\Psi\left(a_{1}, \ldots, a_{N}\right)=\left\langle\chi^{a_{1}}\left(z_{1}\right) \chi^{a_{2}}\left(z_{2}\right) \cdots \chi^{a_{N}}\left(z_{N}\right)\right\rangle
$$

To evaluate $\Psi\left(a_{1}, \ldots, a_{N}\right)$, we use the two-point correlator of Majorana fermions

$$
\left\langle\chi^{a}(z) \chi^{b}(w)\right\rangle=\frac{\delta_{a b}}{z-w}
$$

The multipoint correlators of Majorana fermions are obtained by Wick's theorem

$$
\left\langle\chi^{a}\left(z_{1}\right) \chi^{a}\left(z_{2}\right) \cdots \chi^{a}\left(z_{N_{a}}\right)\right\rangle=\left\{\begin{array}{cc}
\operatorname{Pf}_{a}\left(\frac{1}{z_{i}-z_{j}}\right) & N_{a} \text { even } \\
0 & N_{a} \text { odd }
\end{array}\right.
$$

Therefore, to obtain a nonvanishing $\Psi\left(a_{1}, \ldots, a_{N}\right)$, we must have even $N$. Additionally, $N_{a}$, the number of vectors $\left|n^{a}\right\rangle$ in the spin configuration, must also be even for all $a=1, \ldots, n$.

To compare with the projected BCS wave function, let us evaluate the superposition coefficient of the infinite MPS for a spin configuration, which has $N_{a}$ vector $\left|n^{a}\right\rangle$ at positions $x_{1}^{(a)}<\cdots<x_{N_{a}}^{(a)}\left(N_{a}\right.$ even and $\left.\sum_{a=1}^{n} N_{a}=N\right)$. Taking into account the anticommuting nature of Majorana fermion fields, we first pick up the vectors $\left|n^{1}\right\rangle$ in the spin configuration and rewrite the infinite MPS as

$$
\begin{aligned}
\Psi\left(\left\{x^{(1)}\right\},\left\{x^{(2)}\right\}, \ldots\left\{x^{(n)}\right\}\right)= & \operatorname{sgn}\left(x_{1}^{(1)}, \ldots, x_{N_{1}}^{(2)}, y_{1}, \ldots, y_{N-L_{1}}\right)\left\langle\chi^{a=1}\left(z_{x_{1}^{(1)}}\right) \chi^{a=1}\left(z_{x_{2}^{(1)}}\right) \cdots \chi^{a=1}\left(z_{x_{N_{1}}^{(1)}}\right)\right\rangle \\
& \times\left\langle\chi^{b}\left(z_{y_{1}}\right) \chi^{b}\left(z_{y_{2}}\right) \cdots \chi^{b}\left(z_{y_{N-N_{1}}}\right)\right\rangle \quad(b \neq 1) \\
= & \operatorname{sgn}\left(x_{1}^{(1)}, \ldots, x_{N_{1}}^{(2)}, y_{1}, \ldots, y_{N-L_{1}}\right) \operatorname{Pf}_{a=1}\left(\frac{1}{z_{i}-z_{j}}\right) \\
& \times\left\langle\chi^{b}\left(z_{y_{1}}\right) \chi^{b}\left(z_{y_{2}}\right) \cdots \chi^{b}\left(z_{y_{N-N_{1}}}\right)\right\rangle
\end{aligned}
$$

where the positions $y_{1}<\cdots<y_{N-N_{1}}$ correspond to the vectors $\left|n^{b}\right\rangle$ with $b \neq 1$. The above steps can be repeated from $b=2$ to $n$. In the end, we obtain

$$
\Psi\left(\left\{x^{(1)}\right\},\left\{x^{(2)}\right\}, \ldots\left\{x^{(n)}\right\}\right)=\operatorname{sgn}\left(x_{1}^{(1)}, \ldots, x_{N_{1}}^{(1)}, x_{1}^{(2)}, \ldots, x_{N_{2}}^{(2)}, \ldots, x_{1}^{(n)}, \ldots, x_{N_{n}}^{(n)}\right) \prod_{a=1}^{n} \operatorname{Pf}_{a}\left(\frac{1}{z_{i}-z_{j}}\right)
$$

Comparing with Eq. (1), we conclude that the infinite MPS and the projected BCS state are equivalent.

\section{Derivation of the parent Hamiltonian}

In this Section, we derive the parent Hamiltonian for the infinite MPS.

\section{Brief summary of the $\mathbf{S O}(n)_{1}$ WZW model}

For infinite MPS associated to WZW models, the derivation of the parent Hamiltonian relies on the existence of null vectors in the representation spaces of Kac-Moody algebra [9]. For $\mathrm{SO}(n)_{1}$ WZW model, the Kac-Moody algebra is defined by

$$
\left[J_{n}^{a b}, J_{m}^{c d}\right]=i f^{a b, c d, e f} J_{n+m}^{e f}+n \delta_{a b, c d} \delta_{n+m, 0} \quad n, m \in \mathbb{Z}
$$


where repeated indices are summed over and the $\mathrm{SO}(n)$ structure constant $f^{a b, c d, \text { ef }}$ is given by

$$
f^{a b, c d, e f}=\delta_{a d} \delta_{b e} \delta_{c f}+\delta_{b c} \delta_{a e} \delta_{d f}-\delta_{a c} \delta_{b e} \delta_{d f}-\delta_{b d} \delta_{a e} \delta_{c f}
$$

For odd $n(n \geq 3)$, the $\mathrm{SO}(n)_{1}$ WZW model has three primary fields respectively in singlet (denoted by $I$ ), vector $(v)$ and spinor representation $(s)$, whose conformal weights are $h_{I}=0, h_{v}=1 / 2$ and $h_{s}=n / 16$, respectively. For even $n(n \geq 4)$, apart from the primary fields in singlet and vector representations $\left(h_{I}=0\right.$ and $\left.h_{v}=1 / 2\right)$, the $\mathrm{SO}(n)_{1}$ WZW model has two primary fields in spinor representations (denoted by $s_{+}$and $s_{-}$), whose conformal weights are $h_{s_{+}}=h_{s_{-}}=n / 16$. The $\mathrm{SO}(n)_{1} \mathrm{WZW}$ model has central charge $c=n / 2$ and can be constructed by combining $n$ Ising models $\left(c=n \times \frac{1}{2}\right)$.

For both odd and even $n$, the primary fields in the vector representation have conformal weight $h_{v}=1 / 2$ and are naturally interpreted as Majorana fermions, which are the key ingredients for us to construct the infinite MPS.

For each Majorana fermion $\chi^{a}(a=1, \ldots, n)$, a primary state $\left|\chi^{a}\right\rangle$ can be defined by

$$
\left|\chi^{a}\right\rangle=\chi^{a}(0)|0\rangle
$$

where $|0\rangle$ is the vacuum of the WZW model and satisfies $J_{n>0}^{a b}|0\rangle=0$. When acting on the Kac-Moody currents, the primary states satisfy

$$
\begin{aligned}
& J_{0}^{a b}\left|\chi^{c}\right\rangle=-\sum_{d=1}^{n}\left(L^{a b}\right)_{c d}\left|\chi^{d}\right\rangle \\
& J_{n}^{a b}\left|\chi^{c}\right\rangle=0 \quad(n>0)
\end{aligned}
$$

where $L^{a b}$ are given by

$$
\left(L^{a b}\right)_{c d}=i\left(\delta_{a c} \delta_{b d}-\delta_{b c} \delta_{a d}\right)
$$

Note that $L^{a b}$ form a closed $\mathrm{SO}(n)$ algebra

$$
\begin{aligned}
{\left[L^{a b}, L^{c d}\right] } & =i\left(\delta_{a d} L^{b c}+\delta_{b c} L^{a d}-\delta_{a c} L^{b d}-\delta_{b d} L^{a c}\right) \\
& =i f^{a b, c d, e f} L^{e f}
\end{aligned}
$$

\section{Null vectors and parent Hamiltonian}

To derive the parent Hamiltonian, one has to find the null vectors in the $\mathrm{SO}(n)_{1}$ Kac-Moody algebra. The null vectors are descendant states satisfying

$$
J_{n}^{a b}|\phi\rangle=0 \quad(n>0)
$$

For our purpose, we look for null vectors with the following form:

$$
\left|\phi^{d}\right\rangle=\sum_{a<b, c} W_{a b c}^{d} J_{-1}^{a b}\left|\chi^{c}\right\rangle
$$

where $W_{a b c}^{d}$ are the coefficients that have to be determined. They satisfy the orthonormal condition

$$
\sum_{a<b, c}\left(W_{a b c}^{d^{\prime}}\right)^{*} W_{a b c}^{d}=\delta_{d^{\prime} d}
$$

In general, the tensor $W_{a b c}^{d}$ corresponds to a Clebsch-Gordan decomposition. For the $\mathrm{SU}(2)_{k} \mathrm{WZW}$ model, the $\mathrm{SU}(2)$ Clebsch-Gordan coefficients are known [9]. However, we are not aware of a closed form for the $\mathrm{SO}(n)$ Clebsch-Gordan coefficients. To overcome this difficulty, let us consider the norm of $\left|\phi^{d}\right\rangle$

$$
\begin{aligned}
\left\langle\phi^{d} \mid \phi^{d}\right\rangle & =\sum_{a^{\prime}<b^{\prime}, c^{\prime}} \sum_{a<b, c}\left(W_{a^{\prime} b^{\prime} c^{\prime}}^{d}\right)^{*} W_{a b c}^{d}\left\langle\chi^{c^{\prime}}\left|J_{1}^{a^{\prime} b^{\prime}} J_{-1}^{a b}\right| \chi^{c}\right\rangle \\
& =\sum_{a^{\prime}<b^{\prime}, c^{\prime}} \sum_{a<b, c} W_{a^{\prime} b^{\prime} c^{\prime}}^{d} M_{a^{\prime} b^{\prime} c^{\prime}, a b c} W_{a b c}^{d} \\
& =\left(W^{d}\right)^{\dagger} M W^{d}
\end{aligned}
$$


where $W^{d}$ is viewed as a column vector and $M$ is a matrix defined by

$$
M_{a^{\prime} b^{\prime} c^{\prime}, a b c}=\left\langle\chi^{c^{\prime}}\left|J_{1}^{a^{\prime} b^{\prime}} J_{-1}^{a b}\right| \chi^{c}\right\rangle
$$

If $\left|\phi^{d}\right\rangle$ is a null state, $\left\langle\phi^{d} \mid \phi^{d}\right\rangle=\left(W^{d}\right)^{\dagger} M W^{d}=0$. Since $M$ comes from the norm of two descendent states, it is a positive-semidefinite matrix satisfying $\left(W^{d}\right)^{\dagger} M W^{d} \geq 0$. Therefore, identifying the orthonormal vectors $W^{d}$ that belong to the kernel of $M$ gives us all null states $\left|\phi^{d}\right\rangle$. For our $\mathrm{SO}(n)_{1}$ WZW model, we can write down the explicit form of $M$

$$
\begin{aligned}
M_{a^{\prime} b^{\prime} c^{\prime}, a b c} & =\left\langle\chi^{c^{\prime}}\left|J_{1}^{a^{\prime} b^{\prime}} J_{-1}^{a b}\right| \chi^{c}\right\rangle \\
& =\left\langle\chi^{c^{\prime}}\left|\left[J_{1}^{a^{\prime} b^{\prime}}, J_{-1}^{a b}\right]\right| \chi^{c}\right\rangle \\
& =\left\langle\chi^{c^{\prime}}\left|\left(i \sum_{e f} f^{a^{\prime} b^{\prime}, a b, e f} J_{0}^{e f}+\delta_{a^{\prime} b^{\prime}, a b}\right)\right| \chi^{c}\right\rangle \\
& =\left\langle\chi ^ { c ^ { \prime } } \left|\left(-i \sum_{e f} f^{a^{\prime} b^{\prime}, a b, e f} \sum_{g}\left(L^{e f}\right)_{c g}\left|\chi^{g}\right\rangle+\delta_{a^{\prime} b^{\prime}, a b}\left|\chi^{c}\right\rangle\right)\right.\right. \\
& =-i \sum_{e f} f^{a^{\prime} b^{\prime}, a b, e f}\left(L^{e f}\right)_{c c^{\prime}}+\delta_{a^{\prime} b^{\prime}, a b} \delta_{c^{\prime}, c}
\end{aligned}
$$

where we used Kac-Moody algebra (3) and the properties of the primary state (44). In this way, the null vectors for the $\mathrm{SO}(n)_{1}$ WZW model are obtained.

Let us mention that the above approach is a systematic way of finding null vectors and can be easily generalized to other WZW models. The role of the positive-semidefinite matrix $M$ is similar to the Gram matrix for defining the Kac determinant [15] in conformal field theory.

After obtaining $W_{a b c}^{d}$ for all the null vectors, we define the following $K$ tensor [9]

$$
K_{a^{\prime} b^{\prime}, c^{\prime}}^{a b, c}=\sum_{d}\left(W_{a^{\prime} b^{\prime} c^{\prime}}^{d}\right)^{*} W_{a b c}^{d} \quad\left(a<b \text { and } a^{\prime}<b^{\prime}\right)
$$

Let us write these $K$ tensors as $n \times n$ matrices, $K_{a^{\prime} b^{\prime}, c^{\prime}}^{a b, c}=\left(K_{a^{\prime} b^{\prime}}^{a b}\right)_{c, c^{\prime}}$. Then, $K_{a^{\prime} b^{\prime}}^{a b}$ have the following compact form

$$
K_{a^{\prime} b^{\prime}}^{a b}=\frac{2}{3} \delta_{a b, a^{\prime} b^{\prime}}-\frac{n+2}{6(n-1)} i f^{a b, a^{\prime} b^{\prime}, c d} L^{c d}+\frac{n-4}{6(n-1)}\left(L^{a b} L^{a^{\prime} b^{\prime}}+L^{a^{\prime} b^{\prime}} L^{a b}\right)
$$

Following Ref. [9], we define an operator $\Lambda_{i}^{a b}(1 \leq a<b \leq n)$

$$
\begin{aligned}
\Lambda_{i}^{a b} & =\sum_{j=1(\neq i)}^{N} w_{i j} \sum_{c<d}\left(K^{(i)}\right)_{c d}^{a b} L_{j}^{c d} \\
& =\sum_{j=1(\neq i)}^{N} w_{i j}\left[\frac{2}{3} L_{j}^{a b}-\frac{n+2}{6(n-1)} i f^{a b, c d, e f} L_{i}^{e f} L_{j}^{c d}+\frac{n-4}{6(n-1)}\left(L_{i}^{a b} L_{i}^{c d}+L_{i}^{c d} L_{i}^{a b}\right) L_{j}^{c d}\right] \\
& =\sum_{j=1(\neq i)}^{N} w_{i j}\left[\frac{2}{3} L_{j}^{a b}-\frac{1}{n-1} L_{i}^{a b}\left(\vec{L}_{i} \cdot \vec{L}_{j}\right)+\frac{1}{3}\left(\vec{L}_{i} \cdot \vec{L}_{j}\right) L_{i}^{a b}\right]
\end{aligned}
$$

where $w_{i j} \equiv\left(z_{i}+z_{j}\right) /\left(z_{i}-z_{j}\right)$ and $\vec{L}_{i} \cdot \vec{L}_{j} \equiv \sum_{a<b} L_{i}^{a b} L_{j}^{a b}$. These operators annihilate the infinite MPS, $\Lambda_{i}^{a b}|\Psi\rangle=0$ $\forall i, a, b$. Moreover, the infinite MPS is an $\mathrm{SO}(n)$ singlet and therefore $\sum_{i} L_{i}^{a b}|\Psi\rangle=0 \forall a, b$. Then, an $\mathrm{SO}(n)$ symmetric parent Hamiltonian can be defined by

$$
H=\sum_{i, a<b}\left(\Lambda_{i}^{a b}\right)^{\dagger} \Lambda_{i}^{a b}+J \sum_{a<b}\left(\sum_{i} L_{i}^{a b}\right)^{2}+E_{0} \quad(J \geq 0)
$$

whose ground state is the infinite MPS with energy $E_{0}$. 
In $1 \mathrm{D}$, we use $z_{j}=\exp \left(i \frac{2 \pi}{N} j\right)$ to ensure translational invariance. Choosing $J=2(N-2) / 3$ and $E_{0}=-2(n-$ 1) $N\left(N^{2}-4\right) / 9$, we arrive at the following explicit form of $H$ :

$$
H=-\sum_{i \neq j} w_{i j}^{2}\left[\frac{n+2}{3}\left(\vec{L}_{i} \cdot \vec{L}_{j}\right)+\frac{n-4}{3(n-1)}\left(\vec{L}_{i} \cdot \vec{L}_{j}\right)^{2}\right]-\frac{n-4}{3(n-1)} \sum_{i \neq j \neq k} w_{i j} w_{i k}\left(\vec{L}_{i} \cdot \vec{L}_{j}\right)\left(\vec{L}_{i} \cdot \vec{L}_{k}\right) .
$$

To obtain the above form, the following identities are quite useful

$$
\begin{aligned}
\sum_{a<b} L_{i}^{a b}\left(\vec{L}_{i} \cdot \vec{L}_{j}\right) L_{i}^{a b} & =\vec{L}_{i} \cdot \vec{L}_{j} \quad(i \neq j) \\
\sum_{a<b} L_{i}^{a b}\left(\vec{L}_{i} \cdot \vec{L}_{j}\right)^{2} L_{i}^{a b} & =2(n-1)-(n-2)\left(\vec{L}_{i} \cdot \vec{L}_{j}\right)-\left(\vec{L}_{i} \cdot \vec{L}_{j}\right)^{2} \quad(i \neq j) \\
\sum_{a<b} L_{i}^{a b}\left(\vec{L}_{i} \cdot \vec{L}_{j}\right)\left(\vec{L}_{i} \cdot \vec{L}_{k}\right) L_{i}^{a b} & =2\left(\vec{L}_{j} \cdot \vec{L}_{k}\right)-\left(\vec{L}_{i} \cdot \vec{L}_{k}\right)\left(\vec{L}_{i} \cdot \vec{L}_{j}\right) \quad(i \neq j \neq k)
\end{aligned}
$$

\section{Jastrow and Pfaffian wave functions in Cartan basis}

In this Section, we derive the explicit Jastrow and Pfaffian forms of the wave functions in Cartan basis.

\section{Cartan basis}

Let us first define the Cartan basis.

The $\mathrm{SO}(n)$ algebra is defined by

$$
\left[L^{a b}, L^{c d}\right]=i\left(\delta_{a d} L^{b c}+\delta_{b c} L^{a d}-\delta_{a c} L^{b d}-\delta_{b d} L^{a c}\right)
$$

For $n=2 l$ and $2 l+1$, we can choose at most $l$ (rank of the algebra) mutually commuting generators as $L^{12}, L^{34}, \ldots, L^{2 l-1,2 l}$. In the vector basis, the $\mathrm{SO}(n)$ generators are defined by $L^{a b}=i\left(\left|n^{a}\right\rangle\left\langle n^{b}|-| n^{b}\right\rangle\left\langle n^{a}\right|\right)$ $(1 \leq a<b \leq n)$. Diagonalizing the Cartan generators gives us the following Cartan basis:

$$
\begin{aligned}
|1,0, \ldots\rangle & =\frac{1}{\sqrt{2}}\left(\left|n^{2}\right\rangle+i\left|n^{1}\right\rangle\right) \\
|-1,0, \ldots\rangle= & \frac{1}{\sqrt{2}}\left(\left|n^{2}\right\rangle-i\left|n^{1}\right\rangle\right) \\
|0,1,0, \ldots\rangle= & \frac{1}{\sqrt{2}}\left(\left|n^{4}\right\rangle+i\left|n^{3}\right\rangle\right) \\
|0,-1,0, \ldots\rangle= & \frac{1}{\sqrt{2}}\left(\left|n^{4}\right\rangle-i\left|n^{3}\right\rangle\right) \\
& \vdots \\
|0,0, \ldots, 1\rangle= & \frac{1}{\sqrt{2}}\left(\left|n^{2 l}\right\rangle+i\left|n^{2 l-1}\right\rangle\right) \\
|0,0, \ldots,-1\rangle= & \frac{1}{\sqrt{2}}\left(\left|n^{2 l}\right\rangle-i\left|n^{2 l-1}\right\rangle\right)
\end{aligned}
$$

For $\mathrm{SO}(2 l)$, the above basis is already complete. For $\mathrm{SO}(2 l+1)$, we have an additional vector $\left|n^{2 l+1}\right\rangle$, which is annihilated by all Cartan generators. Thus, we have the following extra vector for $\mathrm{SO}(2 l+1)$ :

$$
|0,0, \ldots, 0\rangle=\left|n^{2 l+1}\right\rangle
$$

Thus, the Cartan basis for $\mathrm{SO}(2 l)$ and $\mathrm{SO}(2 l+1)$ can be compactly written as

$$
\begin{aligned}
\mid 0, \ldots, m_{\alpha} & = \pm 1, \ldots, 0\rangle=\frac{1}{\sqrt{2}}\left(\left|n^{2 \alpha}\right\rangle \pm i\left|n^{2 \alpha-1}\right\rangle\right) \quad(\alpha=1, \ldots, l) \\
|0,0, \ldots, 0\rangle & =\left|n^{2 l+1}\right\rangle
\end{aligned}
$$

Note that $m_{\alpha}$ is the eigenvalue of the Cartan generator $L^{2 \alpha-1,2 \alpha}$. 


\section{Even $n=2 l$}

Now we derive the Jastrow and Pfaffian forms of the $\mathrm{SO}(n)$ wave functions in the Cartan basis. Actually, this goal can be achieved from either the projected BCS form or the infinite MPS form. In the following we use the projected $\mathrm{BCS}$ form to derive the results.

For $\mathrm{SO}(2 l)$, we define the slave-fermion operators in the Cartan basis

$$
\begin{aligned}
c_{m_{1}= \pm 1}^{\dagger}= & \frac{1}{\sqrt{2}}\left(c_{2}^{\dagger} \pm i c_{1}^{\dagger}\right) \\
c_{m_{2}= \pm 1}^{\dagger}= & \frac{1}{\sqrt{2}}\left(c_{4}^{\dagger} \pm i c_{3}^{\dagger}\right) \\
& \vdots \\
c_{m_{l}= \pm 1}^{\dagger}= & \frac{1}{\sqrt{2}}\left(c_{2 l}^{\dagger} \pm i c_{2 l-1}^{\dagger}\right)
\end{aligned}
$$

After changing the basis, the $\mathrm{SO}(2 l)$ valence-bond singlet operator in the projected BCS state is written as

$$
\sum_{a=1}^{2 l} c_{i, a}^{\dagger} c_{j, a}^{\dagger}=\sum_{\alpha=1}^{l}\left(c_{i, m_{\alpha}=1}^{\dagger} c_{j, m_{\alpha}=-1}^{\dagger}+c_{i, m_{\alpha}=-1}^{\dagger} c_{j, m_{\alpha}=1}^{\dagger}\right)
$$

Using the above form, the $\mathrm{SO}(2 l)$ projected BCS state is rewritten as

$$
\begin{aligned}
\left|\Psi_{\mathrm{SO}(2 l)}\right\rangle= & P_{\mathrm{G}} \exp \left(\sum_{i<j} \frac{1}{z_{i}-z_{j}} \sum_{a=1}^{2 l} c_{i, a}^{\dagger} c_{j, a}^{\dagger}\right)|0\rangle \\
= & P_{\mathrm{G}} \exp \left(\sum_{i \neq j} \frac{1}{z_{i}-z_{j}} \sum_{\alpha=1}^{l} c_{i, m_{\alpha}=1}^{\dagger} c_{j, m_{\alpha}=-1}^{\dagger}\right)|0\rangle \\
= & P_{\mathrm{G}} \prod_{\alpha=1}^{l} \prod_{i \neq j}\left(1+\frac{1}{z_{i}-z_{j}} c_{i, m_{\alpha}=1}^{\dagger} c_{j, m_{\alpha}=-1}^{\dagger}\right)|0\rangle \\
= & P_{\mathrm{G}} \prod_{\alpha=1}^{l}\left[\sum_{N_{\alpha}=0}^{N / 2} \sum_{p_{1}^{(\alpha)}<\cdots<p_{N_{\alpha}}^{(\alpha)} q_{1}^{(\alpha)}<\cdots<q_{N_{\alpha}}^{(\alpha)}} \operatorname{det}\left(\frac{1}{z_{i}-z_{j}}\right){ }_{\left(p_{1}^{(\alpha)} \cdots p_{N_{\alpha}}^{(\alpha)}\right),\left(q_{1}^{(\alpha)} \cdots q_{N_{\alpha}}^{(\alpha)}\right)} \sum_{p_{1}^{(\alpha)}, m_{\alpha}=1} c_{q_{1}^{(\alpha)}, m_{\alpha}=-1} \cdots c_{p_{N_{\alpha}}^{(\alpha)}, m_{\alpha}=1}^{\dagger} c_{q_{N_{\alpha}}^{(\alpha)}, m_{\alpha}=-1}^{\dagger}\right]|0\rangle
\end{aligned}
$$

where $\operatorname{det}\left(\frac{1}{z_{i}-z_{j}}\right)_{\left(p_{1}^{(\alpha)} \ldots p_{N_{\alpha}}^{(\alpha)}\right),\left(q_{1}^{(\alpha)} \cdots q_{N_{\alpha}}^{(\alpha)}\right)}$ is the determinant of the $N_{\alpha} \times N_{\alpha}$ Cauchy matrix restricted to the positions of $c_{m_{\alpha}=1}^{\dagger}$ and $c_{m_{\alpha}=-1}^{\dagger}$ fermions. The following useful identity reduces the Cauchy determinant to a product of Jastrow factors:

$$
\operatorname{det}\left(\frac{1}{z_{i}-z_{j}}\right)_{\left(p_{1}^{(\alpha)} \cdots p_{N_{\alpha}}^{(\alpha)}\right),\left(q_{1}^{(\alpha)} \cdots q_{N_{\alpha}}^{(\alpha)}\right)}=(-1)^{\frac{1}{2} N_{\alpha}\left(N_{\alpha}-1\right)} \frac{\prod_{1 \leq i<j \leq N_{\alpha}}\left(z_{p_{i}^{(\alpha)}}-z_{p_{j}^{(\alpha)}}\right)\left(z_{q_{i}^{(\alpha)}}-z_{q_{j}^{(\alpha)}}\right)}{\prod_{1 \leq i, j \leq N_{\alpha}}\left(z_{p_{i}^{(\alpha)}}-z_{q_{j}^{(\alpha)}}\right)}
$$

Note that the sign factor in the Cauchy determinant can be absorbed by rearranging the fermionic operators

$$
\begin{aligned}
& c_{p_{1}^{(\alpha)}, m_{\alpha}=1}^{\dagger} c_{q_{1}^{(\alpha)}, m_{\alpha}=-1}^{\dagger} c_{p_{2}^{(\alpha)}, m_{\alpha}=1}^{\dagger} c_{q_{2}^{(\alpha)}, m_{\alpha}=-1}^{\dagger} \cdots c_{p_{N_{\alpha}}^{(\alpha)}, m_{\alpha}=1}^{\dagger} c_{q_{N_{\alpha}}^{(\alpha)}, m_{\alpha}=-1}^{\dagger} \\
= & (-1)^{\frac{1}{2} N_{\alpha}\left(N_{\alpha}-1\right)}\left(c_{p_{1}^{(\alpha)}, m_{\alpha}=1}^{\dagger} c_{p_{2}^{(\alpha)}, m_{\alpha}=1}^{\dagger} \cdots c_{p_{N_{\alpha}}^{(\alpha)}, m_{\alpha}=1}^{\dagger}\right)\left(c_{q_{1}^{\dagger}, m_{\alpha}=-1}^{(\alpha)} c_{q_{2}^{(\alpha)}, m_{\alpha}=-1}^{\dagger} \cdots c_{q_{N_{\alpha}}^{(\alpha)}, m_{\alpha}=-1}^{\dagger}\right)
\end{aligned}
$$


Therefore, we obtain

$$
\begin{aligned}
\left|\Psi_{\mathrm{SO}(2 l)}\right\rangle= & P_{\mathrm{G}} \prod_{\alpha=1}^{l}\left[\sum_{N_{\alpha}=0}^{N / 2} \sum_{p_{1}^{(\alpha)}<\cdots<p_{N_{\alpha}}^{(\alpha)}} \sum_{q_{1}^{(\alpha)}<\cdots<q_{N_{\alpha}}^{(\alpha)}} \frac{\prod_{1 \leq i<j \leq N_{\alpha}}\left(z_{p_{i}^{(\alpha)}}-z_{p_{j}^{(\alpha)}}\right)\left(z_{q_{i}^{(\alpha)}}-z_{q_{j}^{(\alpha)}}\right)}{\prod_{1 \leq i, j \leq N_{\alpha}}\left(z_{p_{i}^{(\alpha)}}-z_{q_{j}^{(\alpha)}}\right)}\right. \\
& \left.\times\left(c_{p_{1}^{(\alpha)}, m_{\alpha}=1}^{\dagger} c_{p_{2}^{(\alpha)}, m_{\alpha}=1}^{\dagger} \cdots c_{p_{N_{\alpha}}^{(\alpha)}, m_{\alpha}=1}^{\dagger}\right)\left(c_{q_{1}^{(\alpha)}, m_{\alpha}=-1}^{\dagger} c_{q_{2}^{(\alpha)}, m_{\alpha}=-1}^{\dagger} \cdots c_{q_{N_{\alpha}}^{(\alpha)}, m_{\alpha}=-1}^{\dagger}\right)\right]|0\rangle
\end{aligned}
$$

In the next step, we collect the positions $p_{1}^{(\alpha)}<\cdots<p_{N_{\alpha}}^{(\alpha)}$ and $q_{1}^{(\alpha)}<\cdots<q_{N_{\alpha}}^{(\alpha)}$ into a single set $\left\{x^{(\alpha)}\right\}$ with $x_{1}^{(\alpha)}<\cdots<x_{2 N_{\alpha}}^{(\alpha)}$. Then, the Jastrow factors can be written as

$$
\frac{\prod_{1 \leq i<j \leq N_{\alpha}}\left(z_{p_{i}^{(\alpha)}}-z_{p_{j}^{(\alpha)}}\right)\left(z_{q_{i}^{(\alpha)}}-z_{q_{j}^{(\alpha)}}\right)}{\prod_{1 \leq i, j \leq N_{\alpha}}\left(z_{p_{i}^{(\alpha)}}-z_{q_{j}^{(\alpha)}}\right)} \rightarrow \prod_{1 \leq i<j \leq 2 N_{\alpha}}\left(z_{x_{i}^{(\alpha)}}-z_{x_{j}^{(\alpha)}}\right)^{m_{\alpha, i} m_{\alpha, j}}
$$

up to a sign factor. However, the sign factor can again be compensated by rearranging the fermionic operators to the correct order according to $x_{1}^{(\alpha)}<\cdots<x_{2 N_{\alpha}}^{(\alpha)}$.

The last step is to implement the Gutzwiller projection and switch to the spin basis. As a result, we obtain the $\mathrm{SO}(2 l)$ wave function in Cartan basis

$$
\Psi(\{m\})=\rho_{m} \prod_{\alpha=1}^{l} \prod_{i<j}\left(z_{i}-z_{j}\right)^{m_{\alpha, i} m_{\alpha, j}}
$$

where $\rho_{m}=\operatorname{sgn}\left(x_{1}^{(1)}, \ldots, x_{N_{1}}^{(1)}, \ldots, x_{1}^{(l)}, \ldots, x_{N_{l}}^{(l)}\right)$ if $\sum_{i} m_{\alpha, i}=0 \forall \alpha$ and $\rho_{m}=0$ otherwise.

For $n=4$, one can further simplify Eq. (6) and show that it is equivalent to a product of two spin- $1 / 2$ HaldaneShastry states, if the four vectors are interpreted as two spin- $1 / 2$ states.

$$
\text { Odd } n=2 l+1
$$

Comparing to $\mathrm{SO}(2 l)$, we have an additional slave-fermion operators in the Cartan basis for $\mathrm{SO}(2 l+1)$

$$
c_{m=0}^{\dagger}=c_{2 l+1}^{\dagger}
$$

Then, the valence-bond operator for $\mathrm{SO}(2 l+1)$ is expressed as

$$
\sum_{a=1}^{2 l+1} c_{i, a}^{\dagger} c_{j, a}^{\dagger}=c_{i, m=0}^{\dagger} c_{j, m=0}^{\dagger}+\sum_{\alpha=1}^{l}\left(c_{i, m_{\alpha}=1}^{\dagger} c_{j, m_{\alpha}=-1}^{\dagger}+c_{i, m_{\alpha}=-1}^{\dagger} c_{j, m_{\alpha}=1}^{\dagger}\right)
$$

The expansion of the $\mathrm{SO}(2 l+1)$ projected BCS state is given by

$$
\begin{aligned}
& \left|\Psi_{\mathrm{SO}(2 l+1)}\right\rangle=P_{\mathrm{G}} \exp \left(\sum_{i<j} \frac{1}{z_{i}-z_{j}} \sum_{a=1}^{2 l+1} c_{i, a}^{\dagger} c_{j, a}^{\dagger}\right)|0\rangle \\
& =P_{\mathrm{G}} \exp \left(\sum_{i<j} \frac{1}{z_{i}-z_{j}} c_{i, m=0}^{\dagger} c_{j, m=0}^{\dagger}\right) \exp \left(\sum_{i \neq j} \frac{1}{z_{i}-z_{j}} \sum_{\alpha=1}^{l} c_{i, m_{\alpha}=1}^{\dagger} c_{j, m_{\alpha}=-1}^{\dagger}\right)|0\rangle \\
& =P_{\mathrm{G}}\left[\sum_{N_{0}=0}^{N} \sum_{\left(N_{0} \text { even }\right)} \operatorname{Pf}_{x_{1}^{(0)}<\cdots x_{N_{0}}^{(0)}}\left(\frac{1}{z_{i}-z_{j}}\right) c_{x_{1}^{\dagger}, m=0}^{\dagger} \cdots c_{x_{N_{0}}^{(0)}, m=0}^{\dagger}\right] \\
& \times \exp \left(\sum_{i \neq j} \frac{1}{z_{i}-z_{j}} \sum_{\alpha=1}^{l} c_{i, m_{\alpha}=1}^{\dagger} c_{j, m_{\alpha}=-1}^{\dagger}\right)|0\rangle
\end{aligned}
$$


where the positions of the extra fermion $c_{m=0}^{\dagger}$ are labeled by $x_{1}^{(0)}<\cdots<x_{N_{0}}^{(0)}$. The rest of the calculation is similar to the $\mathrm{SO}(2 l)$ case, except for the presence of a Pfaffian factor due to the extra fermionic mode $c_{m=0}^{\dagger}$. After some algebra, we obtain the $\mathrm{SO}(2 l+1)$ wave function in Cartan basis

$$
\Psi(\{m\})=\rho_{m} \operatorname{Pf}_{0}\left(\frac{1}{z_{i}-z_{j}}\right) \prod_{\alpha=1}^{l} \prod_{i<j}\left(z_{i}-z_{j}\right)^{m_{\alpha, i} m_{\alpha, j}}
$$

where $\rho_{m}=\operatorname{sgn}\left(x_{1}^{(0)} \ldots x_{N_{0}}^{(0)}, x_{1}^{(1)}, \ldots, x_{N_{1}}^{(1)}, \ldots, x_{1}^{(l)}, \ldots, x_{N_{l}}^{(l)}\right)$ if $\sum_{i} m_{\alpha, i}=0 \forall \alpha$ and $\rho_{m}=0$ otherwise.

For $n=3$, the signature of the permutation in $\rho_{m}$ is reduced to the Marshall sign in the spin-1 Haldane-Shastry state [9]. 